\title{
One to one resonant double Hopf bifurcation in aeroelastic oscillators with tuned mass dampers
}

\author{
V. Gattulli*, F. Di Fabio, A. Luongo
}

Dipartimento di Ingegneria delle Strutture, delle Acque e del Terreno, Università di L'Aquila, Piazzale E. Pontieri 2 , 67040 Monteluco di Roio (L'Aquila), Italy

\begin{abstract}
The effects of a tuned added mass on the aeroelastic stability of a single degree of freedom bluff body exposed to a steady flow are investigated. The model captures the essential aspects of the behaviour of flexible structures equipped with Tuned Mass Dampers undergoing galloping oscillations. The system exhibits simple as well double Hopf bifurcations, of non-resonant and 1:1 resonant type. Postcritical behaviour of the system in the neighbourhood of the 1:1 resonant type bifurcation is investigated. Employing the Multiple Scale Method, a second order bifurcation equation in the complex amplitude of motion is obtained. Analytical solutions are used to describe the bifurcation scenario in the cases of both undercritical and supercritical aerodynamic behaviour of the bluff body. The effectiveness of the Tuned Mass Damper even in the postcritical range is proved.
\end{abstract}

\section{Introduction}

The stability of aerodynamic oscillators plays an important role for many structures subjected to wind and/or ocean waves [l $\left.\begin{array}{ll}1 & 3\end{array}\right]$. Therefore, mechanical devices added to the structural system to prevent or reduce the magnitude of aeroelastic phenomena are of current research interest [ 4 7].

The concept of damping a structural system by adding a small mass to it dates back to the beginning of the century. Previous studies on tunable mass dampers have set out to optimize mechanical design characteristics in order to increase the critical value at which the dynamic instability phenomenon is triggered [8,9]. However, in order to investigate system performance when the flow velocity exceeds the critical value, an analysis of the postcritical behaviour is needed.

*Corresponding author. Fax: + 39862434548 .

E mail address: gattulli@ing.univaq.it (V. Gattulli). 
Investigations on the system postcritical behaviour have been performed by means of numerical, analytical and experimental methods [10,11]. Nevertheless, in the numerical and experimental tests presented, the dynamical behaviour has been described only for some specific values of the system parameters. Most importantly, the analysis relies on the assumption that the postcritical behaviour of the system can be described referring to an equivalent single degree of freedom (s.d.o.f.) system whose approximate solution has been pursued by the multiple scale method [10] or by the averaging method in [11].

A first study of the system postcritical behaviour as a 2d.o.f. system has been presented by the authors in Ref. [12], with the aim to describe the entire postcritical scenario in the complete parameter-space. In this study, the primary system (PS) and the added mass (TMD) are assumed to posses a s.d.o.f. and to be linear, with the only source of non-linearities arising from the flowstructure interaction. Using a perturbation method, simple and double Hopf bifurcations, occurring at different values of the parameters, have been analyzed. The effectiveness of TMDs has been shown to persist even in the postcritical range, since TMDs generally reduce the amplitude of oscillations in the supercritical case. However, the analysis developed in Ref. [12] was only partial, since it was assumed that (a) a pair of conjugate eigenvalues of the Jacobian matrix is stable (simple Hopf) or (b) the two pairs are both critical but distinct (non-resonant double Hopf). Indeed, due to the tuning between the PS and the TMD, the two eigenvalue pairs are very close to each other, so it is suspected that (a) the stable pair may play some role in the description of the system behaviour and (b) some interaction between the nearly resonant frequencies may occur. This last problem was tentatively addressed by building up a so-called quasi-resonant solution based on the assumption that the Jacobian matrix admits two wellseparated eigenvectors when the frequencies are still very close to each other; however, this hypothesis breaks down when the two frequencies exactly coalesce, since the matrix becomes defective (nilpotent). The quasi-resonant solution revealed qualitative aspects of the motion which cannot be described by the non-resonant solution. It was therefore concluded that a more refined analysis is necessary, in order to describe the neighbourhood of the coalescence point in the parameter space at which a 1:1 resonant double Hopf bifurcation takes place. However, such an analysis is not trivial, since standard perturbation methods do not work when the Jacobian is defective; therefore an adapted procedure must be employed.

In this paper the model presented in Ref. [13] is considered and the postcritical behaviour of the system is analyzed for a Hopf bifurcation in the region of 1:1 resonance. The multiple scale method (MSM) is employed to analyze the defective bifurcation according to the algorithm developed in Ref. [14]. This analysis is believed to be new, both from a mathematical and a physical point of view. A second order complex bifurcation equation in the amplitude of the unique critical mode is derived and the postcritical scenario is analyzed in the bifurcation parameter space. Then, the limits of validity of the concept of equivalent s.d.o.f. introduced in Refs. $[10,11]$ are critically discussed, in the light of the more accurate analysis performed here.

\section{Problem formulation}

An elastically supported bluff body connected with a small added mass and subject to a steady flow is considered (Fig. 1). Both the bluff body primary system (PS) and the added mass (TMD) 


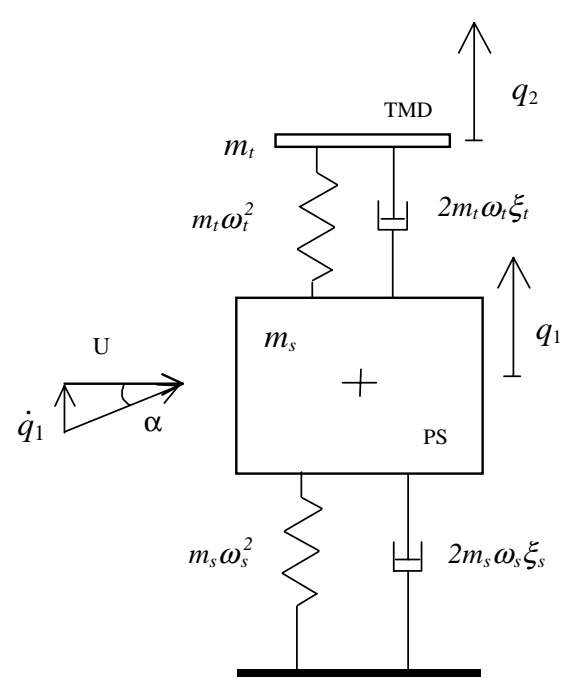

Fig. 1. Aeroelastic oscillator with tuned mass damper.

are assumed to possess a s.d.o.f. and to be linear. The aerodynamic forces acting on the TMD are assumed to be negligible in comparison with those acting on the PS. These forces are obtained using the quasi-static theory [15] taking into account both drag and lift components and retaining the linear and cubic terms of their Taylor expansion. The equation of motion has been derived in Ref. [12]. By using non-dimensional quantities and adopting a state-space representation they read:

$$
\dot{\mathbf{x}}=\mathbf{L x}+\mathbf{f}(\mathbf{x}, \mathbf{m})
$$

In Eq. (1)

$$
\mathbf{L}=\left[\begin{array}{ll}
\mathbf{0} & \mathbf{I} \\
\mathbf{K} & \mathbf{C}
\end{array}\right]
$$

is the system matrix with

$$
\mathbf{C}=\left[\begin{array}{ccc}
2 \xi_{s}(1 & v)+2 \mu \gamma \xi_{t} & 2 \mu \gamma \xi_{t} \\
& 2 \gamma \xi_{t} & 2 \gamma \xi_{t}
\end{array}\right], \quad \mathbf{K}=\left[\begin{array}{cc}
1+\mu \gamma^{2} & \mu \gamma^{2} \\
\gamma^{2} & \gamma^{2}
\end{array}\right]
$$

being the damping and stiffness matrices, respectively; $\mathbf{x}=\left\{q_{1}, q_{2}, \dot{q}_{1}, \dot{q}_{2}\right\}^{\mathrm{T}}$ is the state-space vector, with $q_{1}$ and $q_{2}$ the non-dimensional cross-flow displacements of PS and TMD, respectively; $\mathbf{m}=\left\{\mu, \gamma, \xi_{t}, v\right\}$ is the vector of control parameters; $\mathbf{f}=\left\{f_{i}\right\}$ collects the non-linear part of the vector field, whose components are

$$
f_{1}=0, \quad f_{2}=0, \quad f_{3}=\frac{1}{2} \frac{\delta^{2} A_{1} A_{3}}{\xi_{s} v} x_{3}^{3}, \quad f_{4}=0 .
$$


In Eqs. (1) (4) the following non-dimensional variables have been introduced:

$$
\begin{aligned}
& q_{1}=\frac{\hat{q}_{1}}{D}, \quad q_{2}=\frac{\hat{q}_{2}}{D}, \quad \mu=\frac{m_{t}}{m_{s}}, \quad \gamma=\frac{\omega_{t}}{\omega_{s}}, \quad v=\frac{U}{U_{u n c}}, \\
& U=\frac{\hat{U}}{\omega_{s} D}, \quad U_{u n c}=\frac{2 \xi_{s}}{\delta A_{1}}, \quad \delta=\frac{1}{2} \frac{\rho_{a} D^{2}}{m_{s}}, \quad t=\hat{t} \omega_{s},
\end{aligned}
$$

where $D$ is a typical dimension of the body, $m_{s}, m_{t}, \xi_{s}, \xi_{t}$ are masses and damping coefficients, $\omega_{s}$ and $\omega_{t}$ are the undamped frequencies of the two isolated bodies, $U$ is the uniform flow velocity, $U_{u n c}$ its critical value for the uncontrolled structure; $A_{i}$ are the aerodynamic coefficients, $\rho_{a}$ the air density and $t$ the time, the hat denoting dimensional quantities.

\section{Bifurcation analysis}

The equations of motion (1) admit the equilibrium position $\mathbf{x}=\mathbf{0}$. The position is stable or unstable depending on the values of the parameters $\mathbf{m}$, especially on the parameter $v$ which accounts for the flow velocity. The problem has been analyzed in Refs. [8 11] and completely described in Ref. [12] where analytical expressions of non-resonant and resonant double Hopf manifolds are given. A 3-D representation of the critical manifolds is depicted in Fig. 2a for a fixed value of $\mu$ in the $\left(v, \xi_{t}, \gamma\right)$-space, while the manifolds in the $\left(v, \xi_{t}, \mu\right)$-space can be found in

(a)
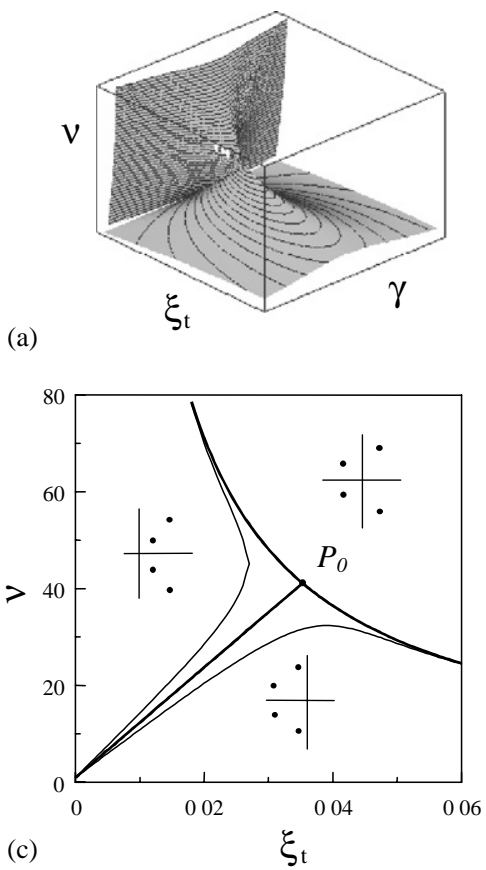

(b)
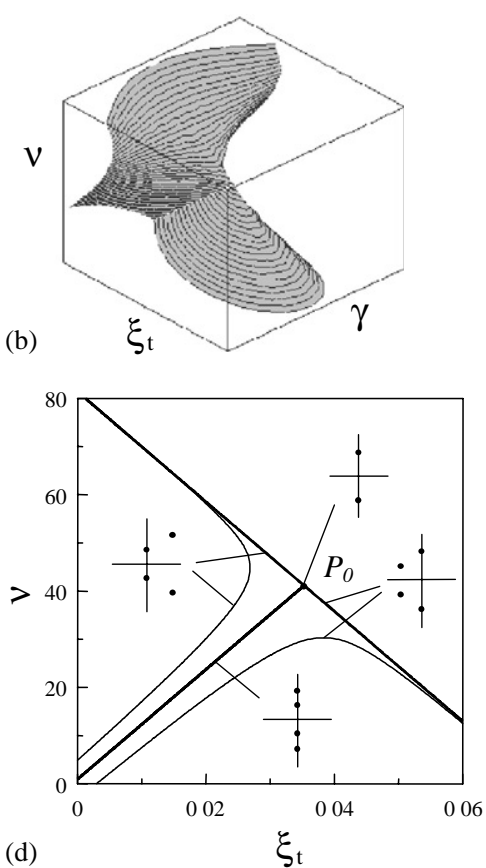

Fig. 2. Critical manifolds in the $\left(v, \xi_{t}, \gamma\right)$ space for $\mu=0.005$; exact (a,c) versus perturbative solution (b,d): (a) and (b) $3 \mathrm{D}$ view; (c) and (d) sections for different $\gamma$ (heavy line $\gamma=\gamma_{0}=0.9975$, light line $\gamma=0.9925$ ). 
Ref. [13]. Highlighted, in particular, is the existence of a peculiar point $P_{0} \equiv\left(v_{0}, \xi_{t 0}, \gamma_{0}\right)$ at which the critical flow velocity is maximized (optimum TMD that realizes a strong enhancement of the critical flow velocity). In Fig. 2c sections at $\gamma=\gamma_{0}$ and $\gamma \neq \gamma_{0}$ of the critical manifold are shown. For a high level of damping $\xi_{t}$ in the TMD, a simple Hopf occurs; at a low level of damping a non-resonant double Hopf bifurcation or two successive closely-spaced bifurcations manifest themselves. At $P_{0}$ a 1:1 resonant double Hopf bifurcation occurs.

The interest is here focused on the system postcritical behaviour around the point $P_{0}$. At this point, the system is defective (or nilpotent) since the critical eigenvalues $\lambda_{1,2}=+\mathrm{i} \omega, \lambda_{3,4}=$ $\mathrm{i} \omega\left(\mathrm{i}=\sqrt{ }\right.$ 1) coalesce into one pair. Only one right eigenvector $\mathbf{u}_{1}$ and one left eigenvector $\mathbf{v}_{2}$ is associated with $\lambda_{1,2}=+\mathrm{i} \omega$, namely:

$$
\left(\mathbf{L}_{0} \quad \mathrm{i} \omega \mathbf{I}\right) \mathbf{u}_{1}=0, \quad\left(\mathbf{L}_{0} \quad \mathrm{i} \omega \mathbf{I}\right)^{\mathrm{H}} \mathbf{v}_{2}=0,
$$

where $\mathbf{L}_{0}$ is the state-space matrix evaluated at $P_{0}$ and $\mathrm{H}$ denotes the transpose conjugate. The defective bases are completed by the generalized $\mathbf{u}_{2}$ and $\mathbf{v}_{1}$ eigenvectors, which are (not unique) solutions of

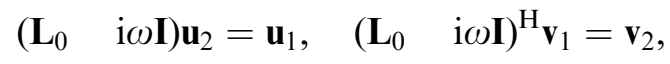

respectively. Right and left eigenvectors are made bi-orthonormal, i.e., $\mathbf{v}_{j}^{\mathrm{H}} \mathbf{u}_{j}=\delta_{i j}(i, j=1,2)$.

Since the bifurcation has codimension-3 (i.e., three conditions of the eigenvalues hold, namely $\left.\operatorname{Re}\left(\lambda_{1}\right)=\operatorname{Re}\left(\lambda_{2}\right)=0, \operatorname{Im}\left(\lambda_{1}\right)=\operatorname{Im}\left(\lambda_{2}\right)\right)$, three bifurcation parameters transverse to the critical manifold must be taken. By fixing $\mu$, the remaining $\left(v, \xi_{t}, \gamma\right)$-parameters are selected as bifurcation parameters and the neighbourhood of $P_{0}$ in Fig. 2 is spanned. The multiple scale method (MSM) is applied to perform the non-linear analysis, according to the procedure illustrated in Ref. [14].

The deviations of the parameters from the bifurcation values $\left(v_{0}, \xi_{t 0}, \gamma_{t 0}\right)$ are assumed to be small, of order $\varepsilon^{2}$, with $\varepsilon$ a perturbation parameter, namely:

$$
v=v_{0}+\varepsilon^{2} v_{2}, \quad \gamma=\gamma_{0}+\varepsilon^{2} \gamma_{2}, \quad \xi_{t}=\xi_{t 0}+\varepsilon^{2} \xi_{t 2} .
$$

The incremental parameter $v_{2}$ represents a distinguished parameter (positive for overcritical flow velocities) and the incremental parameters $\xi_{t 2}$ and $\gamma_{2}$ represent splitting parameters. Moreover, the state-space variables are expanded in series of integer powers of $\varepsilon$ as

$$
\mathbf{x}(t, \varepsilon)=\varepsilon \mathbf{x}_{1}+\varepsilon^{2} \mathbf{x}_{2}+\varepsilon^{3} \mathbf{x}_{3}+\varepsilon^{4} \mathbf{x}_{4}+O\left(\varepsilon^{5}\right)
$$

and several independent temporal scales $t_{k}=\varepsilon^{k} t(k=0,1, \ldots)$ are introduced, so that $\mathrm{d} / \mathrm{d} t=$ $\mathrm{d}_{0}+\varepsilon \mathrm{d}_{1}+\cdots$, with $\mathrm{d}_{k}:=\partial / \partial t_{k}$. By substituting the previous equations in the Eq. (1) and collecting terms with the same powers of $\varepsilon$, the following perturbation equations are drawn up to the $\varepsilon^{4}$-order:

$$
\begin{aligned}
& \left(\begin{array}{ll}
\mathbf{L}_{0} & \mathrm{~d}_{0}
\end{array}\right) \mathbf{x}_{1}=0, \\
& \left(\begin{array}{ll}
\mathbf{L}_{0} & \mathrm{~d}_{0}
\end{array}\right) \mathbf{x}_{2}=\mathrm{d}_{1} \mathbf{x}_{1}, \\
& \left(\begin{array}{ll}
\mathbf{L}_{0} & \mathrm{~d}_{0}
\end{array}\right) \mathbf{x}_{3}=\mathrm{d}_{1} \mathbf{x}_{2}+\left(\begin{array}{lll}
\mathrm{d}_{2} & \mathbf{L}_{2}
\end{array}\right) \mathbf{x}_{1} \quad \frac{1}{6} \mathbf{f}_{\mathbf{x x x}}^{0} \mathbf{x}_{1}^{3}, \\
& \left(\begin{array}{ll}
\mathbf{L}_{0} & \mathrm{~d}_{0}
\end{array}\right) \mathbf{x}_{4}=\mathrm{d}_{1} \mathbf{x}_{3}+\left(\begin{array}{lll}
\mathrm{d}_{2} & \mathbf{L}_{2}
\end{array}\right) \mathbf{x}_{2} \quad \frac{1}{2} \mathbf{f}_{\mathbf{x x x}}^{0} \mathbf{x}_{1}^{2} \mathbf{x}_{2} .
\end{aligned}
$$


In Eqs. (10) $\mathbf{L}_{2}$ is the second order part of the $\varepsilon$-expansion of $\mathbf{L}$ around $P_{0}$ (i.e., $\mathbf{L}=\mathbf{L}_{0}+\varepsilon^{2} \mathbf{L}_{2}$ ), whose submatrices, according to Eqs. (2) and (3), are

$$
\begin{aligned}
& \mathbf{C}_{2}=2\left[\begin{array}{cc}
\xi_{s} \nu_{2}+\mu\left(\gamma_{0} \xi_{t 2}+\gamma_{2} \xi_{t 0}\right) & \mu\left(\gamma_{0} \xi_{t 2}+\gamma_{2} \xi_{t 0}\right) \\
\left(\gamma_{0} \xi_{t 2}+\gamma_{2} \xi_{t 0}\right) & \left(\gamma_{0} \xi_{t 2}+\gamma_{2} \xi_{t 0}\right)
\end{array}\right], \\
& \mathbf{K}_{2}=2\left[\begin{array}{cc}
\mu \gamma_{0} \gamma_{2} & \mu \gamma_{0} \gamma_{2} \\
\gamma_{0} \gamma_{2} & \gamma_{0} \gamma_{2}
\end{array}\right] .
\end{aligned}
$$

Finally, $\mathbf{f}_{\mathbf{x x x}}^{0}$ is the third derivative of the non-linear part of the vector field at $P_{0}$, namely

$$
\mathbf{f}_{\mathbf{x x x}}^{0} \mathbf{x y z}=6 \delta \frac{A_{3}}{U} x_{3} y_{3} z_{3} .
$$

It should be noted that, in Eq. (12), the actual value $U$ of the flow velocity is used, instead of the bifurcation value $U_{0}$ [2]. Although this procedure is inconsistent, numerical results have shown that it improves the accuracy of the solution for $U \gg U_{0}[12]$.

According to the spectral properties (6) of the defective matrix $\mathbf{L}_{0}$, the non-diverging generating solution of Eq. (10) reads

$$
\mathbf{x}_{1}=A \mathbf{u}_{1} \mathrm{e}^{\mathrm{i} \omega t_{0}}+\text { c.c. },
$$

where $A$ is the complex amplitude depending on slower time scales and c.c. stands for the complex conjugate terms. By substituting Eq. (13) in Eq. (10) and accounting for Eq. (7), it follows that

$$
\mathbf{x}_{2}=\mathrm{d}_{1} A \mathbf{u}_{2} \mathrm{e}^{\mathrm{i} \omega t_{0}}+\text { c.c. }
$$

It should be noted that, although $L_{0}$ is singular, no solvability conditions must be enforced on Eq. $\left(10_{2}\right)$, since the known term belongs to the range of the operator. In contrast, starting on $\varepsilon^{3}$ order perturbation equations, solvability requires that the known term be orthogonal to the unique left eigenvector $\mathbf{v}_{2}$. By accounting for Eq. (13), the solvability of Eq. $\left(10_{3}\right)$ reads

$$
\mathbf{v}_{2}^{\mathrm{H}}\left(\mathrm{d}_{1}^{2} A \mathbf{u}_{2} \quad A \mathbf{L}_{2} \mathbf{u}_{1} \quad \frac{1}{2} A^{2} A \mathbf{f}_{\mathbf{x x x}}^{0} \mathbf{u}_{1}^{2} \mathbf{u}_{1}\right)=0,
$$

where an overbar denotes the complex conjugate. From Eq. (15) an ordinary differential equation in the complex amplitude $A\left(t_{1}, t_{2}, \ldots\right)$ follows:

$$
\mathrm{d}_{1}^{2} A=s_{31} A+s_{32} A^{2} A
$$

whose coefficients (and those introduced from now on) are given in Appendix A. By solving Eq. (10), and omitting the complementary function, it is found that

$$
\mathbf{x}_{3}=\left(\begin{array}{lll}
\mathrm{d}_{2} A \mathbf{u}_{2} & p_{31} A \mathbf{u}_{2} & p_{32} A^{2} A \mathbf{u}_{2}
\end{array}\right) \mathrm{e}^{\mathrm{i} \omega t_{0}}+\mathbf{z}_{111} A^{3} \mathrm{e}^{3 \mathrm{i} \omega t_{0}} .
$$

By using Eqs. (14) and (17) in $\left(10_{4}\right)$ the relevant solvability conditions read

$$
2 \mathrm{~d}_{1} \mathrm{~d}_{2} A=s_{41} \mathrm{~d}_{1} A+s_{42} A A \mathrm{~d}_{1} A+s_{43} A^{2} \mathrm{~d}_{1} A .
$$

The solvability conditions in Eqs. (16) and (18) are combined in a unique equation by coming back to the true time $t$ through a consistent reconstitution procedure [16]. By using the chain rule

$$
\frac{\mathrm{d}^{2} A}{\mathrm{~d} t^{2}}=\left(\varepsilon^{2} \mathrm{~d}_{1}^{2}+2 \varepsilon^{3} \mathrm{~d}_{1} \mathrm{~d}_{2}\right) A+O\left(\varepsilon^{4}\right)
$$


the evolutive equation for the postcritical complex amplitude $A$ follows:

$$
\frac{\mathrm{d}^{2} A}{\mathrm{~d} t^{2}}=C_{1} A+C_{2} \frac{\mathrm{d} A}{\mathrm{~d} t}+C_{3} A^{2} A+C_{4} A A \frac{\mathrm{d} A}{\mathrm{~d} t}+C_{5} A^{2} \frac{\mathrm{d} A}{\mathrm{~d} t} .
$$

In Eq. (20) the parameter $\varepsilon$ has been absorbed in accordance with $\varepsilon A \rightarrow A, \varepsilon \mathrm{d} / \mathrm{d} t \rightarrow \mathrm{d} / \mathrm{d} t$, and the coefficients $C_{i}$ are reported in Appendix A. Expressing the amplitudes in polar form $A=\frac{1}{2} a(t) \mathrm{e}^{\mathrm{i} \theta(t)}$ and separating the real and imaginary parts of Eqs. (20), four differential equations of the first order in the real variables $(a, \theta, r, s)$ follow:

$$
\begin{aligned}
& \dot{a}=r, \\
& \dot{r}=R_{1} a+a s^{2}+\frac{1}{4} R_{3} a^{3}+R_{2} r \quad I_{2} a s+\frac{1}{4}\left(R_{4}+R_{5}\right) a^{2} r+\frac{1}{4}\left(I_{5} \quad I_{4}\right) a^{3} s, \\
& a \dot{s}=I_{1} a \quad 2 r s+\frac{1}{4} I_{3} a^{3}+R_{2} a s+I_{2} r+\frac{1}{4}\left(I_{4}+I_{5}\right) a^{2} r+\frac{1}{4}\left(R_{4} \quad R_{5}\right) a^{3} s, \\
& \dot{\theta}=s,
\end{aligned}
$$

where $R_{i}=\operatorname{Re}\left(C_{i}\right)$ and $I_{i}=\operatorname{Im}\left(C_{i}\right)$. In Eqs. $\left(21_{1}\right)\left(21_{3}\right)$, the unknown variables $(a, r, s)$, are in number equal to the codimension of the problem; the variables describe the postcritical behaviour of the system of Eq. (1) in the region of a 1:1 resonant double Hopf bifurcation. Eq. (214), decoupled from the previous equations, describes the evolution of the phase $\theta$. The steady state solutions of (21) are obtained by zeroing the right-hand side terms of $\left(21_{1}\right)\left(21_{3}\right)$. Since a cubic equation in $a^{2}$ can be drawn, up to three real non-trivial solutions $(a, s)$ are sought, depending on the control parameter values. The solutions represent periodic motion (limit cycles) of system (1) with constant amplitude $a$ and frequency $\Omega=\omega+s$.

\section{Bifurcation scenario}

Numerical investigations have been carried out to analyze the system postcritical behaviour using the illustrated analytical solutions as well as direct time-integration of the equations of motion. Use is made of either Eq. (20) in the complex amplitude $A$ or its equivalent real representation given by Eqs. (21).

Eqs. (20) and (21) admit the trivial solution $A=0 \forall t$. In order to evaluate the region of the parameters where a non-trivial solution exists, a bifurcation analysis is performed. Since Eq. (21) is in non-standard normal form (in particular Eq. $\left(21_{3}\right)$ contains the product $a \dot{s}$ ) the standard Jacobian eigenvalue analysis fails. Therefore, the complex amplitude equation must be directly discussed.

Considering only the linear part of Eq. (20), it can be re-written in a state-space form as

$$
\left[\begin{array}{l}
\dot{A} \\
\ddot{A}
\end{array}\right]=\left[\begin{array}{cc}
0 & 1 \\
C_{1} & C_{2}
\end{array}\right]\left[\begin{array}{l}
A \\
\dot{A}
\end{array}\right],
$$

where both $A$ and $C_{i}$ are complex quantities. In particular, $\operatorname{Re}\left(C_{i}\right)$ and $\operatorname{Im}\left(C_{i}\right)$ are linear combinations of the $\left(v_{2}, \xi_{t 2}, \gamma_{2}\right)$-parameters through the matrix $\mathbf{L}_{2}$ (see Appendix A). The stability 
of the trivial solution $A=0$ of Eq. (20) is governed by the spectrum of the complex state matrix in Eq. (22). Its eigenvalues are given by

$$
\lambda_{i}=\frac{1}{2}\left(C_{2} \pm \sqrt{ } C_{2}^{2}+4 C_{1}\right), \quad i=1,2
$$

and the critical condition at which a static bifurcation takes place is $\operatorname{Re}\left(\lambda_{i}\right)=0$. By expressing the coefficients $C_{i}$ in terms of the $\left(v_{2}, \xi_{t 2}, \gamma_{2}\right)$-parameters, and using Eqs. (8) the critical conditions furnish two manifolds, represented in Fig. 2b. They are tangent at $P_{0}$ to the exact manifolds of Fig. 2a, evaluated through the spectral analysis of the linear part of Eq. (1) (see also Ref. [12]), and therefore represent a local approximation of these manifolds. The section at $\gamma=\gamma_{0}$ and $\gamma \neq \gamma_{0}$ illustrated in Fig. 2d, and compared with Fig. 2c, shows the degree of approximation achieved by the $\varepsilon^{4}$-order expansion.

The postcritical behaviour is described by analyzing the dependence of the limit-cycle amplitudes on the control parameters. Fig. 3 shows the amplitude $a$ versus the flow velocity $v$ and the damping $\xi_{t}$ for the perfect tuned system $\left(\gamma=\gamma_{0}\right)$ and supercritical aerodynamic behaviour $\left(A_{3}<0\right.$ in Eq. (4)). The regions of existence of the limit cycles are depicted in Fig. 3a, namely: in the region $\mathscr{R}_{0}$ no limit cycles exist, but only the stable trivial solution is admitted; in $\mathscr{R}_{1}$ and $\mathscr{R}_{2}$ and $\mathscr{R}_{3}$ one, two, and three limit cycles exist, respectively, according to the discussion of Eqs. (21). Such solutions are represented in a 3 -D-view in Fig. 3 b. The two stable solutions of region $\mathscr{R}_{2}$ emerge from the double Hopf boundary $S_{2}$; they coalesce in the amplitude $a$ but differ in the frequency correction $s$. A unique solution rises from the simple Hopf boundaries, being stable at $S_{1}^{+}$(positive velocity of the eigenvalues at the criticality) and unstable at $S_{1}$ (negative velocity). The three solutions existing in the region $\mathscr{R}_{3}$ coalesce along the line $\mathscr{C}$.

In Fig. $3 b$ four sections are selected, three (I, II, III) parallel to the $(v, a)$-plane, the fourth (VI) parallel to the $\left(\xi_{t}, a\right)$-plane. Path I crosses the region $\mathscr{R}_{0}, \mathscr{R}_{2}$ and $\mathscr{R}_{3}$ showing the occurrence of a successive bifurcation at $S_{1}$. Path II contains the peculiar point $P_{0}$ and it shows the transition from the region $\mathscr{R}_{0}$ to the line $\mathscr{C}$, where the three solutions coalesce in one. Path III illustrates the passage from $\mathscr{R}_{0}$ to $\mathscr{R}_{1}$ through a simple Hopf bifurcation. Path IV explains the coalescence mechanism: the two stable solutions of $\mathscr{R}_{2}$ are associated to almost opposite frequency corrections (see $s$ in Fig. 3e) while the unstable solution has no frequency correction. At the crossing between path IV and the line $\mathscr{C}$, the three solutions coalesce to one with zero frequency correction (Figs. $3 \mathrm{~d}$ and e). It is worth noticing that the coalescence occurs at the smallest amplitude existing for any given $v>v_{0}$.

The analytical results of Fig. 3 have been compared with the direct numerical integration of the equations of motion (1) and an excellent accordance between them has been found (see Figs. $3 \mathrm{c}$ and d). Moreover, the curves have been compared with that of the uncontrolled system, which only depends on $v$. It is seen that the limit-cycle amplitudes of the controlled system are always below the uncontrolled ones. Therefore, the TMD has a beneficial effect even in the postcritical range, the maximum benefit occurring at $v=v_{0}$, i.e., at the optimum value of the TMD.

To better illustrate the role of the frequency correction $s$, the two limit cycles existing at the point $Q \in \mathscr{R}_{2}$, with the same amplitudes and opposite corrections, have been depicted on the $\left(q_{1}, q_{2}\right)$-configuration plane (Fig. 4). Here, the perturbative solution at the first order $\left(\mathbf{x}=\varepsilon \mathbf{x}_{1}\right.$, I-curves) and that at the second order $\left(\mathbf{x}=\varepsilon \mathbf{x}_{1}+\varepsilon^{2} \mathbf{x}_{2}\right.$, II-curves $)$ have been compared with direct 


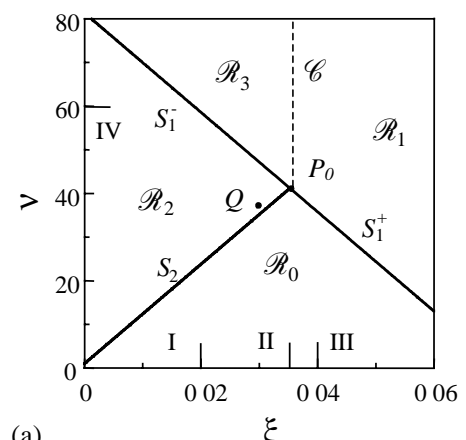

(a)

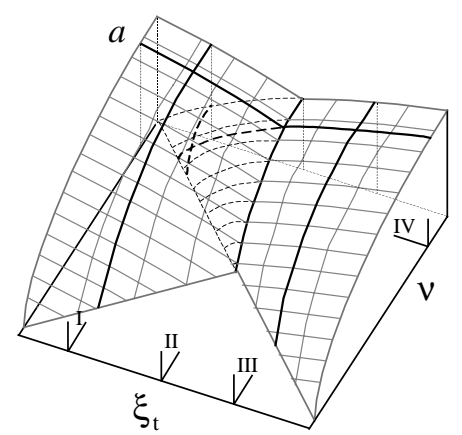

(b)
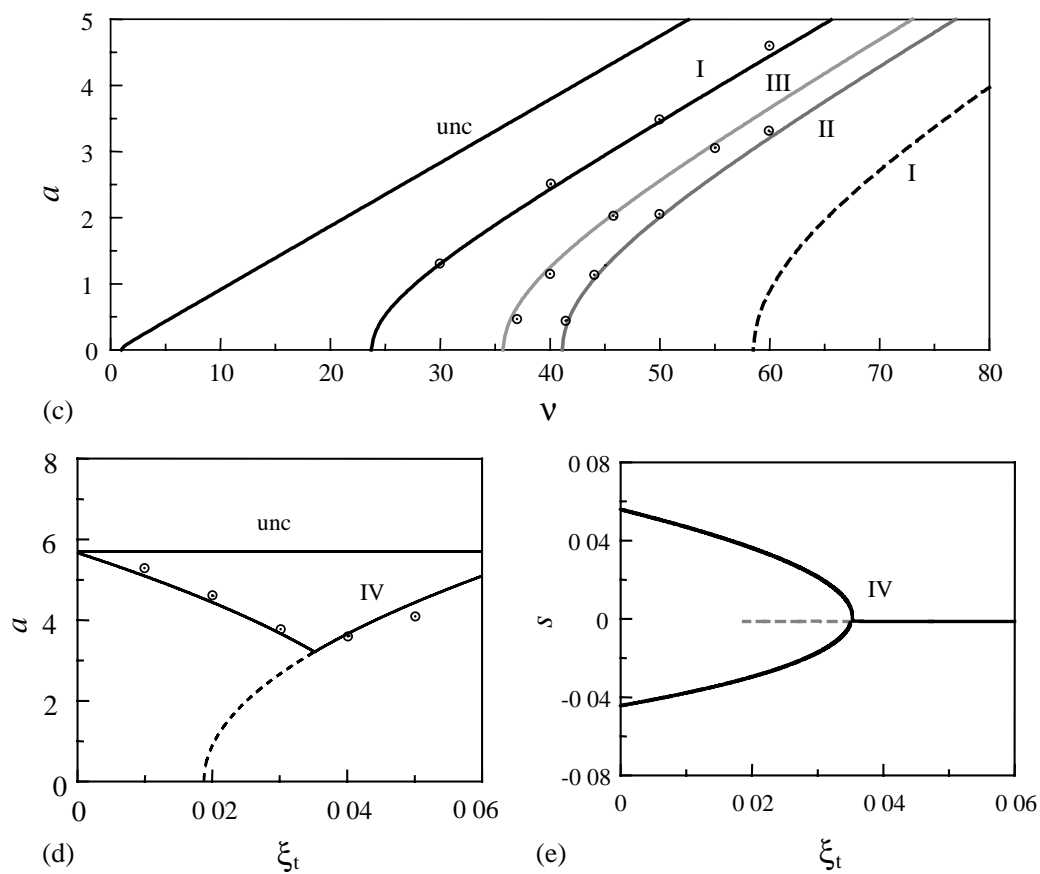

Fig. 3. Supercritical scenario in the $\left(v, \xi_{t}\right)$ space for $\gamma=\gamma_{0}=0.9975$ and $A_{3}=421$ : (a) existence regions of non trivial solutions, (b) $3 \mathrm{D}$ view in the $\left(v, \xi_{t}, a\right)$ space, (c) I, II, III $(v, a)$ sections, (d) IV $\left(\xi_{t}, a\right)$ section, (e) IV $\left(\xi_{t}, s\right)$ section. Continuous lines: stable solutions; dashed lines: unstable solutions; dots: numerical results; unc: uncontrolled system.

numerical integration (EX-curves). The first order solution appears quite rough in comparison with the second order solution, which is very close to the exact one. The differences are ascribed to the fact that the first order solution $\mathbf{x}_{1}$ (Eq. (13)), predicts steady state oscillations along the proper eigenvector $\mathbf{u}_{1}$ at $P_{0}$; therefore, it is not able to capture the dependence of the $q_{1} / q_{2}$ ratio on the control parameters. The second order term $\mathbf{x}_{2}$ (Eq. (14)) instead accounts for such modification, since its factor $\mathrm{d}_{1} A=\mathrm{i} s A$ is parameter-dependent through the frequency correction s. Therefore the second order solution $\mathbf{x} \simeq \varepsilon \mathbf{x}_{1}+\varepsilon^{2} \mathbf{x}_{2}=A\left(\mathbf{u}_{1}+\mathrm{i} s \mathbf{u}_{2}\right) \mathrm{e}^{\mathrm{i} \omega t}+$ c.c. is an harmonic motion along a vector that is just the lower order approximation of the proper eigenvectors at a 

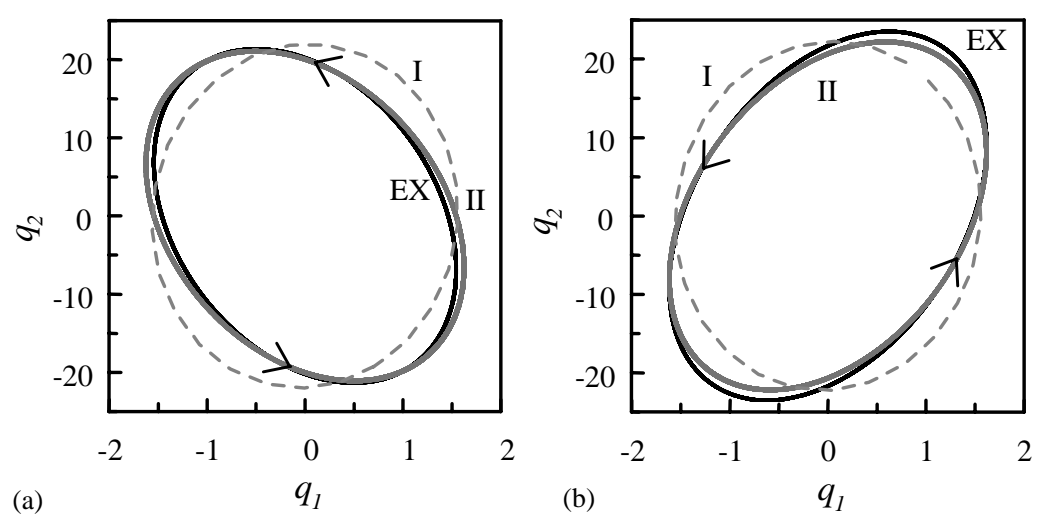

Fig. 4. Limit cycle at point $Q \equiv(37.8,0.033,0.9975)$ in the $\left(q_{1} ; q_{2}\right)$ configuration plane; direct integration (EX) versus I order and II order perturbative solutions: (a) $s>0$; (b) $s<0$.

$Q_{i}$-point [17], if non-linearities are neglected in $s$. However, since the sensitivity of the eigenvectors of a defective matrix is high, it is expected that the region of validity of the resonant solution is quite limited.

The effect on the postcritical behaviour of imperfect tuning $\left(\gamma \neq \gamma_{0}\right)$ between the PS and the TMD is analyzed in Fig. 5. The regions in which the limit cycles exist are illustrated in Fig. 5a. The simultaneous passage of two eigenvalues across the imaginary axis is broken by the detuning (it should be remembered that the locus of double Hopf belongs to the plane $\gamma=\gamma_{0}$ ); as a consequence, a simple Hopf occurs for increasing $v$ for any value of $\xi_{t}$ along the lower boundary $S_{1 l}^{+}$. From it a regular surface arises, as illustrated in Fig. 5b. Along the higher boundaries $S_{1 h}^{+}$and $S_{1}$, two successive simple Hopfs occur, with positive and negative velocities of the eigenvalues respectively. There, new surfaces arise, which coalesce along the line $\mathscr{C}$, where a locus of limit points with respect to $\xi_{t}$ occurs. In summary, with respect to the perfectly tuned case (Fig. 3b) the two surfaces in $\mathscr{R}_{2}$ split: the upper one smoothly matches the surface in $\mathscr{R}_{1}$, while the lower one matches the surface in $\mathscr{R}_{3}$. Two typical sections (I, II in Fig. $5 \mathrm{~b}$ ) are sufficient to describe the scenario for increasing $v$ (see Fig. 5c). Path I crosses all the regions $\mathscr{R}_{i}(i=0, \ldots, 3)$. Along it a stable limit cycle first bifurcates from the trivial solution at $S_{1 l}^{+}$; then an initially unstable limit cycle bifurcates at $S_{1 h}^{+}$; finally, at $S_{1}$, a third unstable limit cycle bifurcates while the second one regains stability through a Neimark bifurcation, from which modulated solutions arise (not studied here). Path II corresponds to a simple Hopf bifurcation with a stable limit cycle. Path III shows the dependence of the amplitude (Fig. 5a) and of the frequency correction (Fig. 5e) on $\xi_{t}$. The curves can be usefully interpreted as perturbations of those in Figs. $3 \mathrm{~d}$ and e, due to the detuning. The separation of the coincident solutions appears clearly. Along the lower branch in Fig. $5 \mathrm{~d}$ first a Neimark bifurcation at $S_{1}$ and then a limit point at $\mathscr{C}$ occur. Finally, the $s$-path of Fig. $5 \mathrm{~d}$ shows that the detuning gives rise to the lack of symmetry of the two solutions in $\mathscr{R}_{2}$. Consequently, these solutions are represented by two orbits in the $\left(q_{1}, q_{2}\right)$-configuration plane with different amplitudes and orientations. Direct numerical integration of Eq. (1) shows a complete qualitative agreement (see Figs. $5 \mathrm{c}$ and d), while quantitative differences increase when moving away from the point $P_{0}$. Moreover, the amplitudes are found to be always below that of 

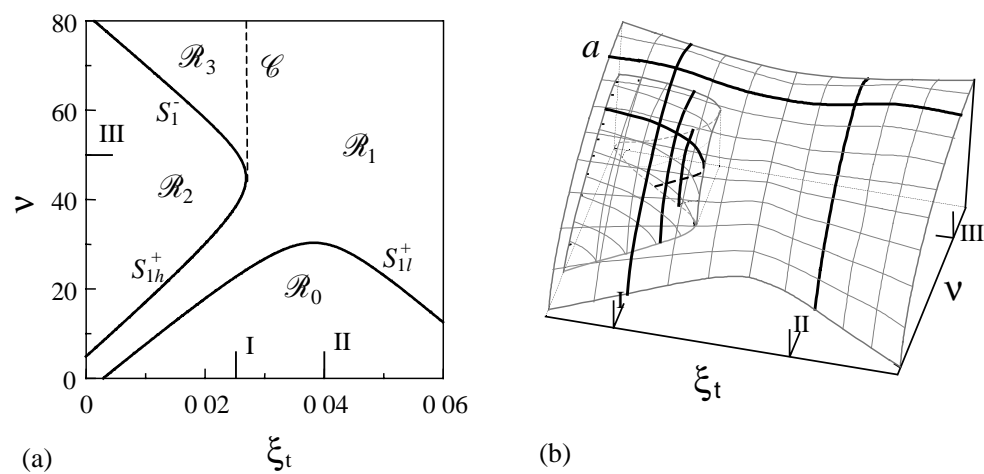

(b)

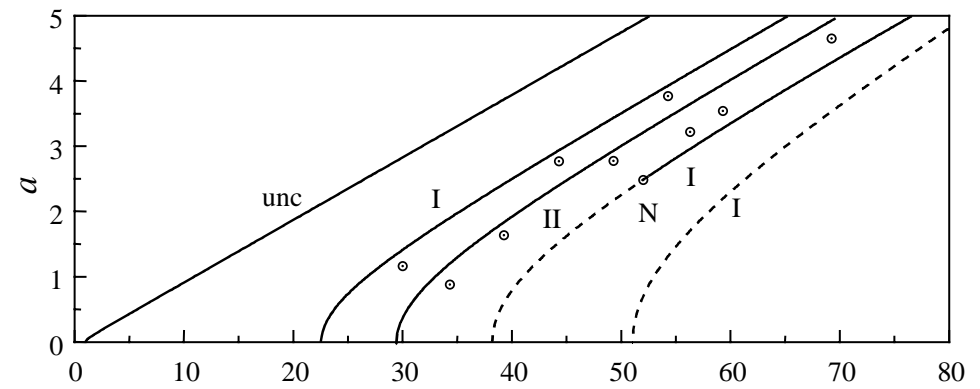

(c)

$\mathrm{V}$
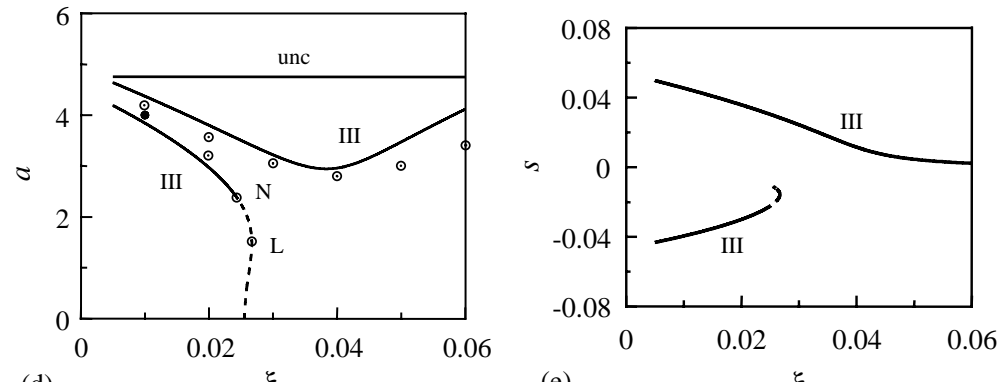

(d) $\xi_{\mathrm{t}}$

(e)

$\xi_{\mathrm{t}}$

Fig. 5. Supercritical scenario in the $\left(v, \xi_{t}\right)$ plane for $\gamma=0.9925$ and $A_{3}=421$ : (a) existence regions of non trivial solutions, (b) $3 \mathrm{D}$ view in the $\left(v, \xi_{t}, a\right)$ space; (c) I, II $(v, a)$ sections; (d) III $\left(\xi_{t}, a\right)$ section, (e) III $\left(\xi_{t}, s\right)$ section. Continuous lines: stable solutions; dashed lines: unstable solutions; dots: numerical results; unc: uncontrolled system.

the uncontrolled system, except for a very small $\xi_{t}$ not shown in Fig. $5 \mathrm{~d}$. This erroneous result is however a consequence of the rough approximation of the bifurcation locus $S_{1 l}^{+}$far from $P_{0}$ given by the perturbation solution, as is apparent from Figs. $2 \mathrm{c}$ and $\mathrm{d}$.

The influence of the sign of the non-linear aerodynamical force on system postcritical behaviour is then investigated. A positive aerodynamic coefficient $A_{3}$ is considered (i.e., a section with a subcritical aerodynamic behaviour) and the previous analysis is repeated. In the perfectly tuned case $\left(\gamma=\gamma_{0}\right)$ the scenario changes as seen in Fig. 6. In Fig. 6a the regions of limit cycle existence are illustrated; they are of course bounded by the same manifolds $S_{1}^{+}, S_{1}, S_{2}$ of the supercritical 


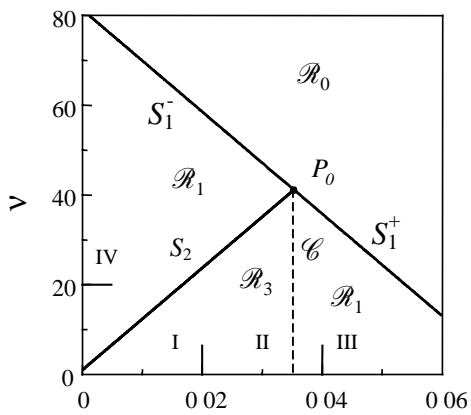

(a)
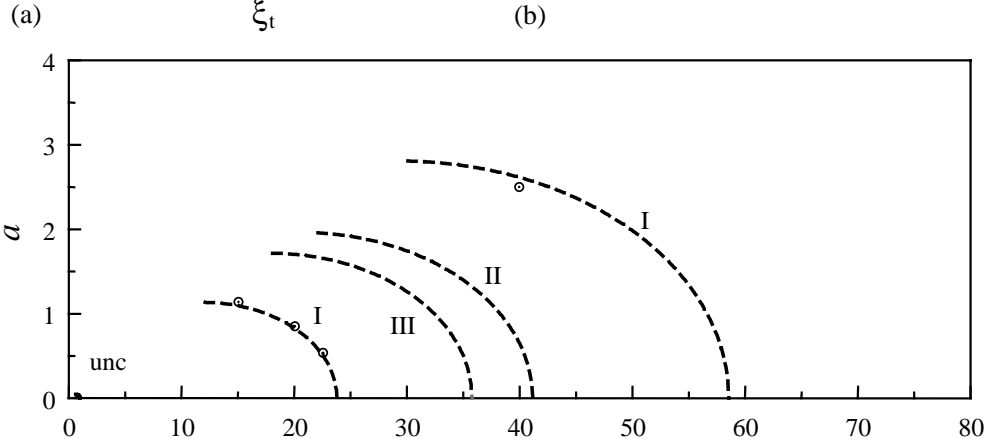

(c)
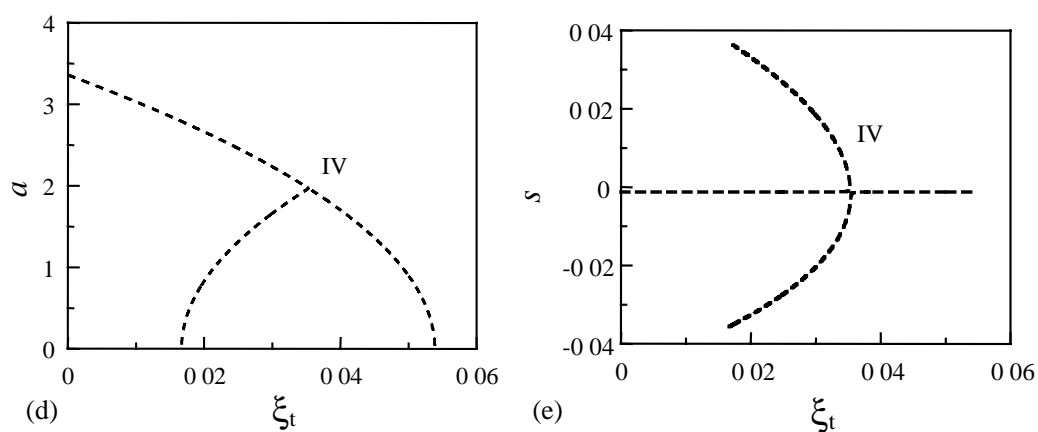

Fig. 6. Subcritical scenario in the $\left(v, \xi_{t}\right)$ plane for $\gamma=\gamma_{0}=0.9975$ and $A_{3}=421$ : (a) existence regions of non trivial solutions, (b) $3 \mathrm{D}$ view in the $\left(v, \xi_{t}, a\right)$ space; (c) I, II and III $(v, a)$ sections; (d) IV $\left(\xi_{t}, a\right)$ section, (e) IV $\left(\xi_{t}, s\right)$ section. Continuous lines: stable solutions; dashed lines: unstable solutions; dots: numerical results; unc: uncontrolled system.

case. In $\mathscr{R}_{0}$, i.e., for high wind velocities, no limit cycles exist (see Fig. 6b); at $S_{1}^{+}$and $S_{1}$ a subcritical limit cycle surface emerges; at $S_{2}$ a subcritical surface of two limit cycles with the same amplitude and opposite frequency correction arise and coalesce with the previous one at the line $\mathscr{C}$; therefore no region with two limit cycles exists. All the solutions are found to be unstable. Sections I III are illustrated in Fig. 6c; Section IV in Fig. 6d. Along path I and for decreasing wind velocities $v$, no stable solutions exist in $\mathscr{R}_{0}$ so that the motion diverges. The larger-amplitude unstable solution defines the attraction basin of the equilibrium position in region $\mathscr{R}_{1}$, while the 


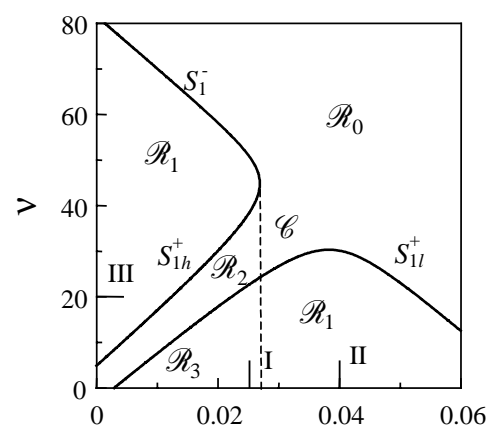

(a)

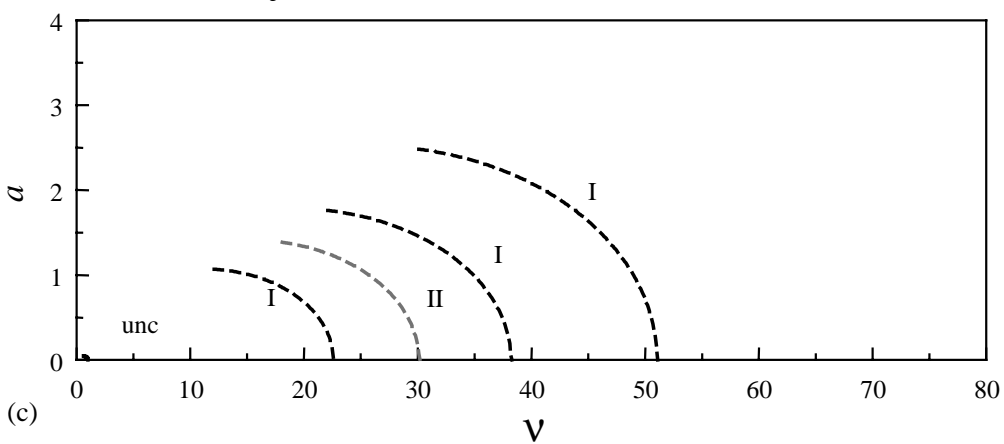

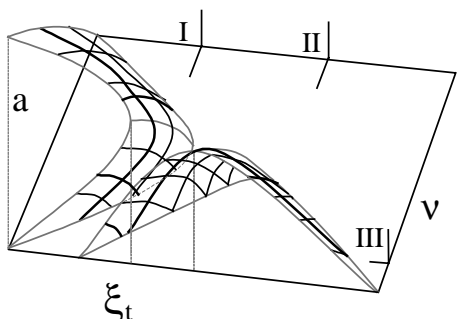

(b)

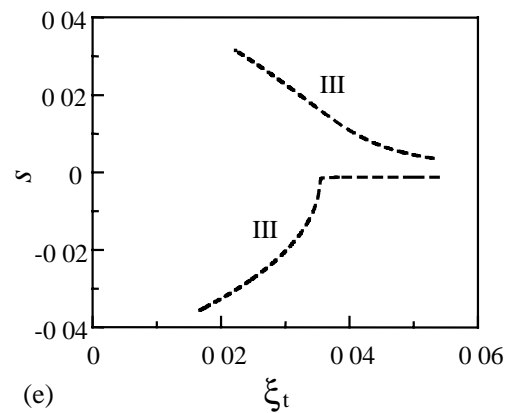

Fig. 7. Subcritical scenario in the $\left(v, \xi_{t}\right)$ plane for $\gamma=0.9925$ and $A_{3}=421$ : (a) existence regions of non trivial solutions, (b) $3 \mathrm{D}$ view in the $\left(v, \xi_{t}, a\right)$ space; (c) I, II $(v, a)$ sections; (d) III $\left(\xi_{t}, a\right)$ section, (e) III ( $\left.\xi_{t}, s\right)$ section. Continuous lines: stable solutions; dashed lines: unstable solutions; dots: numerical results; unc: uncontrolled system.

smaller solution bounds that in $\mathscr{R}_{3}$. Path II follows the locus $\mathscr{C}$ of the amplitude-coalescing solutions (with no frequency corrections). Path III illustrates the Hopf subcritical bifurcation while path IV shows the coalescence mechanism (Figs. 6a and e). Numerical results have again confirmed the perturbation analysis (Fig. 6c). Finally, the amplitude curves, compared with those of the uncontrolled system (where they are almost unnoticeable in the scale of the plot) turn out to be all higher: therefore the TMD has a beneficial effect also on sections with subcritical behaviour, since it enlarges the attraction basin. 
In Fig. 7 analysis is completed with subcritical aerodynamic behaviour in the imperfectly tuned case. The results should be read as a perturbation of the tuned case.

\section{Discussion}

Some results have been presented in literature $[10,11]$ to demonstrate the effectiveness of the TMD also in the postcritical range. They are based on the assumption that the steady dynamics of a PS equipped with a TMD can be described by an equivalent s.d.o.f. system. Two different approaches have been followed in those papers. An equivalent structural damping was defined in Ref. [11] by equating the critical velocities of the controlled and uncontrolled systems. According to this criterion, the TMD would only modify the linear properties of the PS, by increasing the structural damping, without affecting the non-linear forces acting on it. A more refined analysis was performed in Ref. [10], by assuming that the ratio between the PS and TMD amplitudes of motion remains constant in time. The procedure leads to an equivalent s.d.o.f. system, in which, again, only the linear terms (damping as well stiffness) are modified by the TMD. In addition, a non-linear algebraic equation must be solved to get the unknown frequency as function of the linear parameters only (mass ratio, frequency ratio and damping). Both equivalent oscillators furnish limit cycle amplitudes smaller than the uncontrolled ones.

The analysis developed in the present paper permits the results of Refs. [10,11] to be discussed. It should initially be observed that the reduction of the system to a s.d.o.f. system is generally incorrect. Indeed, while the non-linear dynamics of the simple aeroelastic oscillator is essentially governed by a one-dimensional equation of the type $\dot{a}=f(a)$, where $a$ is the oscillation amplitude, the dynamics of the coupled system is governed by a three-dimensional equation $\dot{\mathbf{a}}=\mathbf{f}(\mathbf{a})$, where $\dot{\mathbf{a}}=(\dot{a}, a, s)$ and $s$ is the time-dependent frequency correction (see Eq. (21)). This dynamic richness is a direct consequence of the fact that, around the optimum point $P_{0}$, both the pairs of eigenvalues play an active role in the postcritical behaviour, either in the region $\mathscr{R}_{2}$ (where both are unstable) and in region $\mathscr{R}_{1}$ and $\mathscr{R}_{3}$ (where only one is unstable but, the other is close to it). Therefore, no equivalence can exist among systems whose essential dynamics develop in spaces of different dimensions. For example, steady quasi-periodic solutions are admitted by the threedimensional system (although they have been found here to be of unstable type), but are forbidden in the one-dimensional one. The main difference between the two systems is that, while the frequency correction does not substantially affect the dynamics of the simple aeroelastic oscillator (since it does not modify the amplitude of the motion), in contrast it actively contributes to the dynamics of the coupled oscillator (being strongly connected with the amplitude).

From Eq. $\left(21_{3}\right)$ it is seen that, even in the steady motion in which $\dot{\mathbf{a}}=\mathbf{0}$, the frequency correction $s$ depends on both linear and non-linear terms in the amplitude $a$. The linear terms account for the modification occurring in the frequency when the parameters are varied from their optimum value (i.e., they describe the sensitivity of the eigenvalues of the system at the double Hopf bifurcation point $P_{0}$ ); the non-linear terms account for the effects of the aerodynamic forces. The perturbation analysis developed in Section 3 shows that these effects all appear at the same $\varepsilon^{4}$ order (see Eq. (18)) so that it is not allowed to neglect the latter in comparison with the former. If non-linear effects have to be ignored, then, consistently, linear terms must be ignored too, and $s=0$ must be taken, as predicted by the $\varepsilon^{3}$-order perturbation equation (16). 
However, the role played by non-linearities in the frequency correction equation $\left(21_{3}\right)$ is fundamental to a correct description of the system behaviour. Indeed, if they are consistently accounted for, the algebraic problem associated with Eqs. $\left(21_{1,2,3}\right)$ leads to a degree-three equation in the squared amplitude $a$, responsible for the multiple branch solution displayed in regions $\mathscr{R}_{2}$ and $\mathscr{R}_{3}$ (see Figs. 3 and 5). In contrast, if $s$ is taken as independent of the amplitude (e.g., equal to zero), then a linear equation in the squared amplitude is found, structurally indentical to that of the aeroelastic oscillator. Thus, the existence of a region of multiple branch solution is obscured. As an example, if region $\mathscr{R}_{1}$ (with $\gamma=\gamma_{0}$ ) in Fig. 3 is considered (simple Hopf bifurcation for the perfectly tuned system), $s=0$ is found, i.e., linear and non-linear effects on frequency balance each other. The following approximate expression for the limit cycle can be derived:

$$
a^{2}=\frac{4}{3} \frac{\left[\left(\xi_{s} v_{2}+\xi_{t 2}\right) / \mu\right] U}{\delta A_{3}}=\frac{4}{3} \frac{\xi_{e} U}{\delta A_{3}}
$$

from which an equivalent damping $\xi_{e}$ is drawn. Both the analyses developed in Refs. [10,11] do not account for non-linear frequency correction and, in fact, do not highlight the existence of multiple branch solutions. However, these analyses were applied to systems some way from the double Hopf bifurcation point and only in the region $\mathscr{R}_{1}$, where it is reasonable to suppose that the interaction between the two couples of eigenvalues is weak (since the stable couple has a passive role). Therefore, the conclusions of Refs. [10,11] about the effectiveness of the TMD are correct, but cannot be considered of general validity; however, they have been confirmed and generalized by the wider analysis performed here.

\section{Conclusions}

The postcritical behaviour of a s.d.o.f. system equipped with a Tuned Mass Damper has been analyzed for double Hopf bifurcation in the neighbourhood of 1:1 resonance. Due to the coalescence of its eigenvalues, the system is defective at the criticality, and therefore admits an incomplete set of eigenvectors. By using the Multiple Scale Method, a second order bifurcation equation governing the time-evolution of the complex amplitude of the critical mode has been derived. When a real-variables representation is adopted, three first order differential equations, uncoupled from the fourth and describing the asymptotic dynamic of the system, have been found. By solving the associate algebraic equations, steady solutions have been found representing limit cycles for the original mechanical system. The regions of existence of such limit cycles have been studied in the space of the control parameters. Up to three limit cycles have been found to coexist, both for aerodynamically stable and for aerodynamically unstable section shapes. Perturbation results have been found to be in excellent agreement with results obtained by directly integrating the equations of motion. In all cases considered it has been found that the TMD has a beneficial effect on the postcritical behaviour of the system, since it reduces the limit cycle amplitudes in the supercritical case and increases them in the subcritical case. These results, while they confirm the findings of Refs. [10,11], extend them to the whole parameter region of technical interest, where previous methods of analysis cannot be employed. 


\section{Appendix A}

The expressions of the coefficients in Eqs. (16) (20) are

$$
\begin{gathered}
s_{31}=C_{1}=\mathbf{v}_{2}^{\mathrm{H}} \mathbf{L}_{2} \mathbf{u}_{1}, \\
p_{31}=\mathbf{v}_{1}^{\mathrm{H}} \mathbf{L}_{2} \mathbf{u}_{1}, \\
p_{32}=\frac{1}{2} \mathbf{v}_{1}^{\mathrm{H}} \mathbf{f}_{\mathbf{x x x}}^{0} \mathbf{u}_{1}^{2} \mathbf{u}_{1}, \\
s_{41}=C_{2}=p_{31}+\mathbf{v}_{2}^{\mathrm{H}} \mathbf{L}_{2} \mathbf{u}_{2}, \\
s_{32}=C_{3}=\frac{1}{2} \mathbf{v}_{2}^{\mathrm{H}} \mathbf{f}_{\mathbf{x x x}}^{0} \mathbf{u}_{1}^{2} \mathbf{u}_{1}, \\
s_{42}=C_{4}=2 p_{32} \mathbf{v}_{2}^{\mathrm{H}} \mathbf{u}_{2}+\mathbf{v}_{2}^{\mathrm{H}} \mathbf{f}_{\mathbf{x} \mathbf{x}}^{0} \mathbf{u}_{1} \mathbf{u}_{1} \mathbf{u}_{2}, \\
s_{43}=C_{5}=p_{32} \mathbf{v}_{2}^{\mathrm{H}} \mathbf{u}_{2}+\frac{1}{2} \mathbf{v}_{2}^{\mathrm{H}} \mathbf{f}_{\mathbf{x x x}}^{0} \mathbf{u}_{1}^{2} \mathbf{u}_{2} .
\end{gathered}
$$

In Eqs. (A.1), $\mathbf{u}_{k}$ and $\mathbf{v}_{k}(k=1,2)$ are the right and the left eigenvectors defined by Eqs. (6) and (7); an overbar denotes the complex conjugate and ()$^{\mathrm{H}}$ the transpose conjugate. Moreover, $\mathbf{z}_{111}$ is the solution to the following algebraic problem:

$$
\left(\mathbf{L}_{0} \quad 3 \mathrm{i} \omega_{0}\right) \mathbf{z}_{111}=\frac{1}{6} \mathbf{f}_{\mathbf{x x x}}^{0} \mathbf{u}_{1}^{3} .
$$

\section{References}

[1] G.V. Parkinson, N.P.H. Brooks, On the aeroelastic instability of bluff cylinders, Journal of Applied Mechanics 28 (1961) 252258.

[2] M. Novak, Aeroelastic galloping of prismatic bodies, Engineering of Mechanics Division 96 (1969) 115142.

[3] R.M. Corless, G.V. Parkinson, A model of the combined effects of vortex induced oscillation and galloping, Journal of Fluids and Structures 2 (1988) 203220.

[4] T.T. Soong, G.F. Dargush, Passive Energy Dissipation Systems in Structural Engineering, Wiley, New York, 1997.

[5] A. Larsen, Vortex induced response of bridges and control by tuned mass dampers, in: Moan et al. (Eds.), Structural Dynamics EURODYN'93, A.A. Balkema, Rotterdam, 1993, pp. 10031010.

[6] A. Larsen, E. Svensson, H. Andersen, Design aspects of tuned mass dampers for the Great Belt East Bridge approach span, Journal of Wind Engineering and Industrial Aerodynamics 5455 (1993) 413426.

[7] V. Gattulli, R. Ghanem, Adaptive control of flow induced oscillations including vortex effects, International Journal of Non Linear Mechanics 34 (1999) 853868.

[8] M.D. Rowbottom, The optimization of mechanical dampers to control self excited galloping oscillations, Journal of Sound and Vibration 75 (1981) 559576.

[9] Y. Fujino, M. Abé, Design formulas for tuned mass dampers based on a perturbation technique, Earthquake Engineering and Structural Dynamics 22 (1993) 833854.

[10] Y. Fujino, P. Warnitchai, M. Ito, Suppression of galloping of bridge tower using tuned mass damper, Journal of the Faculty of Engineering, The University of Tokyo 38 (1985) 4973.

[11] M. Abdel Rohman, Design of tuned mass dampers for suppression of galloping in tall prismatic structures, Journal of Sound and Vibration 171 (1994) 289299.

[12] V. Gattulli, F. Di Fabio, A. Luongo, Simple and double Hopf bifurcations in aeroelastic oscillators with tuned mass dampers, Journal of Franklin Institute 338 (2001) 187201. 
[13] A. Luongo, V. Gattulli, F. Di Fabio, 1:1 Resonant Hopf bifurcations in slender space structures with tuned mass dampers, 42nd AIAA/ASME/ASCE/AHS/ASC Structures, Structural Dynamics and Materials Conference, AIAA 2001-1308, 2001.

[14] A. Di Egidio, A. Paolone, A. Luongo, Analisi postcritica di strutture autoeccitate in risonanza 1:1, Proceedings of 14th Italian Conference on Theoretical and Applied Mechanics, AIMETA'99, Como, Italy, 1999.

[15] R.D. Blevins, Flow-Induced Vibration, 2nd Edition, Van Nostrand Reinhold, New York, 1990.

[16] A. Luongo, A. Paolone, On the reconstitution problem in the multiple time-scale method, Nonlinear Dynamics 19 (1999) 133-156.

[17] A. Luongo, Eigensolutions sensitivity for nonsymmetric matrices with repeated eigenvalues, American Institute of Aeronautics and Astronautics Journal 31 (1993) 1321-1328. 